\title{
Infinitesimals and Pavelka logic
}

\author{
Esko Turunen $^{1}$ Mirko Navara ${ }^{2}$ \\ ${ }^{1}$ Tampere University of Technology, Finland \\ ${ }^{2}$ Czech Technical University in Prague, Czech Republic
}

\begin{abstract}
Rational Pavelka Logic does not admit infinitesimals. We argue that infinitesimals are important in logic and we present an alternative approach which admits them. It is built up in a similar style, but based on the Chang's perfect MV-algebra. We prove a partial result towards the completeness of this logic. We also discuss a combined approach using more complex perfect MV-algebras.
\end{abstract}

Keywords: Mathematical fuzzy logic, Rational Pavelka Logic, Łukasiewicz operations, MValgebra, perfect MV-algebra, Chang's MV-algebra.

\section{Aim and organization of the paper}

In Section 2, we introduce MV-algebras as the semantics of all logics considered here. Emphasis is put on perfect MV-algebras and Chang's MValgebra. In Section 3, we summarize Rational Pavelka Logic. In Section 4, we describe our alternative, Perfect Pavelka Logic, whose semantics uses Chang's MV-algebra instead of the standard MValgebra. We prove a partial result about its completeness. In Section 5, we discuss the differences of the two approaches. Both have interpretation in simulation of human reasoning and each of them is advantageous in some situations. We propose a combination of these two principles (based on perfect MV-algebras more complex than the Chang's algebra) as a perspective new direction of research. This could describe more of the human-like logical deduction.

\section{Perfect MV-algebras}

Let us recall some basic definitions and properties of MV-algebras; see [4, 21] for details. An $M V$-algebra $\mathbf{L}=\left\langle L, \oplus,{ }^{*}, \mathbf{0}\right\rangle$ is a structure such that $\langle L, \oplus, \mathbf{0}\rangle$ is a commutative monoid and

$$
\begin{aligned}
x^{* *} & =x, \\
x \oplus \mathbf{0}^{*} & =\mathbf{0}^{*}, \\
\left(x^{*} \oplus y\right)^{*} \oplus y & =\left(y^{*} \oplus x\right)^{*} \oplus x .
\end{aligned}
$$

Denote $x \odot y=\left(x^{*} \oplus y^{*}\right)^{*}$ and $\mathbf{1}=\mathbf{0}^{*}$. Then $\langle L, \odot, \mathbf{1}\rangle$ is also a commutative monoid and $\left\langle\oplus,{ }^{*}, \odot\right\rangle$ is a De Morgan triple. A partial order on the set $L$ is introduced by

$$
x \leq y \text { iff } x^{*} \oplus y=\mathbf{1} \text { iff } x \odot y^{*}=\mathbf{0} .
$$

By setting

$$
\begin{aligned}
& x \vee y=\left(x^{*} \oplus y\right)^{*} \oplus y \\
& x \wedge y=\left(x^{*} \vee y^{*}\right)^{*}\left[=\left(x^{*} \odot y\right)^{*} \odot y\right]
\end{aligned}
$$

for all $x, y, z \in L$, the structure $\langle L, \wedge, \vee\rangle$ is a lattice and $\left\langle\wedge,{ }^{*}, \vee\right\rangle$ is a De Morgan triple. By stipulating

$$
x \rightarrow y=x^{*} \oplus y,
$$

the structure $\langle L, \leq \wedge, \vee, \odot, \rightarrow, \mathbf{0}, \mathbf{1}\rangle$ is a residuated lattice with the bottom and top elements $\mathbf{0 , 1}$, respectively. In particular, a residuation (sometimes also called Galois connection)

$$
x \odot y \leq z \text { iff } x \leq y \rightarrow z
$$

holds for all $x, y, z \in L$. The couple $\langle\odot, \rightarrow\rangle$ is an adjoint couple.

As an example, the Lukasiewicz structure (also called the standard $M V$-algebra) $\mathcal{C} L$ is the real unit interval $[0,1]$ equipped with the usual order and, for each $x, y \in[0,1]$,

$$
\begin{aligned}
x \oplus y & =\min \{x+y, 1\}, \\
x^{*} & =1-x .
\end{aligned}
$$

Moreover,

$$
\begin{aligned}
x \odot y & =\max \{0, x+y-1\} \\
x \vee y & =\max \{x, y\}, \\
x \wedge y & =\min \{x, y\} \\
x \rightarrow y & =\min \{1,1-x+y\} .
\end{aligned}
$$

For any natural number $m \geq 2$, the finite chain $0<\frac{1}{m}<\cdots<\frac{m-1}{m}<1$ can be viewed as an MV-algebra where $\frac{n}{m} \oplus \frac{k}{m}=\min \left\{\frac{n+k}{m}, 1\right\}$ and $\left(\frac{n}{m}\right)^{*}=\frac{m-n}{m}$. Finally, a structure $\mathcal{C} L \cap \mathbf{Q}$ with the Łukasiewicz operations is an example of a countable MV-algebra called rational Eukasiewicz structure. All these examples are MV-subalgebras of the standard MV-algebra $\mathcal{C} L$.

An MV-algebra $L$ is called complete if $\bigvee \Gamma, \bigwedge \Gamma \in$ $L$ for any subset $\Gamma \subseteq L$. The rational Łukasiewicz structure is not complete. Assume $x$ is an element of an MV-algebra $L$ and $\left\{y_{i}\right\}_{i \in \Gamma} \subseteq L$. Then

$$
\begin{aligned}
& x \rightarrow \bigvee_{i \in \Gamma} y_{i}=\bigvee_{i \in \Gamma}\left(x \rightarrow y_{i}\right), \\
& \bigwedge_{i \in \Gamma} y_{i} \rightarrow x=\bigvee_{i \in \Gamma}\left(y_{i} \rightarrow x\right),
\end{aligned}
$$


holds whenever the suprema and infima exist in $L$.

An MV-algebra called Chang's MV-algebra, introduced originally in [3], is obtained by considering the following set $\mathcal{C} C$ of formal symbols:

$$
\begin{aligned}
& \mathbf{0}, c, 2 c, 3 c, \cdots, n c, \cdots \\
& \cdots, 1-n c, \cdots, 1-3 c, 1-2 c, 1-c, \mathbf{1}
\end{aligned}
$$

and then defining the MV-operations as follows (cf. [6])

$$
\begin{aligned}
& \text { if } \quad x=n c \text { and } y=m c, \\
& \text { then } x \oplus y:=(n+m) c, \\
& \text { if } \quad x=1-n c \text { and } y=1-m c, \\
& \text { then } x \oplus y:=\mathbf{1}, \\
& \text { if } \quad x=n c \text { and } y=1-m c \text { and } m \leq n, \\
& \\
& \text { then } x \oplus y:=\mathbf{1}, \\
& \text { if } \quad x=n c \text { and } y=1-m c \text { and } n<m, \\
& \\
& \text { then } x \oplus y:=1-(m-n) c, \\
& \text { if } \quad x=1-m c \text { and } y=n c \text { and } m \leq n, \\
& \\
& \text { then } x \oplus y:=\mathbf{1}, \\
& \text { if } \quad x=1-m c \text { and } y=n c \text { and } n<m, \\
& \\
& \text { then } x \oplus y:=1-(m-n) c, \\
& \text { if } \quad x=n c, \\
& \\
& \text { then } x^{*}:=1-n c, \\
& \text { if } \quad x=1-n c, \\
& \\
& \text { then } x^{*}:=n c .
\end{aligned}
$$

We construct Chang's MV-algebra in another way. Recall [13] that a Product algebra $P$ is a BL-algebra which satisfies additional conditions

$$
\begin{aligned}
x^{* *} & \leq(y \odot x \rightarrow z \odot x) \rightarrow(y \odot z), \\
x \wedge x^{*} & =\mathbf{0}
\end{aligned}
$$

for all $x, y, z \in P$. A simple example is based on the product t-norm $\odot$ on the real unit interval $[0,1]$; $x \odot y=x y$.

Fix an element $t \in P, 0<t<1$. Then the set $T=\left\{t^{n} \mid n \geq 0\right\}$ is an infinite decreasing chain

$$
\cdots<t^{n}<\cdots<t^{3}<t^{2}<t<t^{0}=\mathbf{1} .
$$

Now reverse the order and rename the elements $t^{n}$ by $f^{n}$ as follows

$$
\mathbf{0}=f^{0}<f<f^{2}<f^{3}<\cdots<f^{n}<\cdots
$$

Then the set $F=\left\{f^{n} \mid n \geq 0\right\}$ is an infinite increasing chain. Assuming $f^{n}<t^{n}$ for any natural $n \geq 0$, we construct the set $F \cup T$

$$
\begin{aligned}
& \mathbf{0}<f<f^{2}<f^{3}<\cdots<f^{n}<\cdots \\
& \cdots<t^{n}<\cdots<t^{3}<t^{2}<t<\mathbf{1} .
\end{aligned}
$$

(Here the superscripts of $t, f$ only index these elements, they do not mean any type of power, repeated multiplication or $\odot$-operation.) Notice that
$F \cap T=\emptyset$ and $F \cup T$ is a lattice that is not complete as $\bigvee F$ and $\bigwedge T$ do not exists in $F \cup T$. However, if a supremum, resp. infimum, of a subset of the set $F \cup T$ exists, then it is the greatest, resp. smallest, element of this subset (and conversely). Similarly, we define the operations $\oplus$ and ${ }^{*}$ on $F \cup T$ as follows: for any $m, n \geq 0,\left(f^{n}\right)^{*}=t^{n},\left(t^{n}\right)^{*}=f^{n}$. Moreover,

$$
\begin{aligned}
f^{m} \oplus f^{n} & =f^{m+n}, \\
t^{m} \oplus t^{n} & =\mathbf{1}, \\
f^{m} \oplus t^{n} & = \begin{cases}t^{n-m} & \text { if } n>m, \\
\mathbf{1} & \text { otherwise. }\end{cases}
\end{aligned}
$$

The product operation $\odot$ obeys dual equations

$$
\begin{aligned}
t^{m} \odot t^{n} & =t^{m+n}, \\
f^{m} \odot f^{n} & =\mathbf{0}, \\
t^{m} \odot f^{n} & = \begin{cases}f^{n-m} & \text { if } n>m, \\
\mathbf{0} & \text { otherwise. }\end{cases}
\end{aligned}
$$

Setting $\mathcal{C} C=F \cup T$, we obtain an MV-algebra that is isomorphic to Chang's MV-algebra. The MValgebra $\mathcal{C} C$ is a prototypical example of a perfect $\mathrm{MV}$-algebra; any element $c \in \mathcal{C} C$ satisfies the equation

$$
(c \oplus c) \odot(c \oplus c)=(c \odot c) \oplus(c \odot c) .
$$

We will need the following obvious result

REMARK 1 If $a \neq \mathbf{1}, b \neq \mathbf{0}$ are elements of a linearly ordered $M V$-algebra (in particular, Chang's $M V$-algebra), then $a \oplus b>a$.

The set $F$ is the radical $\operatorname{Rad}(\mathcal{C} C)$ of $\mathcal{C} C$, that is, the intersection of all maximal ideals of $\mathcal{C} C$, and the elements of $F$ are called infinitesimals. In our approach they will be interpreted as falsehood to a degree $f^{n}$, therefore the set is denoted by $F$. Similarly, the set $T$ is the coradical $\operatorname{Rad}(\mathcal{C} C)^{*}$ of $\mathcal{C} C$, the set of the elements of $\mathcal{C} C$ whose complements are infinitesimals. They will be interpreted as truth to a degree $t^{n}$. Notice that the sets $F$ and $T$ are closed with respect to the MV-operations $\odot, \oplus ; T$ even with respect to the operation $\rightarrow$.

\section{Pavelka's Fuzzy Logic}

\subsection{Introduction}

A conventional approach in mathematical sentential logic is first to introduce atomic formulae, logical connectives and the set of well-formed formulae; these formulae are semantically interpreted in suitable algebraic structures. In Classical Logic these structures are Boolean algebras, in Hájek's Basic Fuzzy Logic [13], for example, the suitable structures are BL-algebras. Tautologies of a logic are those formulae that obtain the top value $\mathbf{1}$ in all interpretations in all suitable algebraic structures; for this reason tautologies are sometimes called 1-tautologies to distinguish them from possible weaker notions of tautologies in fuzzy logics. 
For example, tautologies in Basic Fuzzy Logic are exactly the formulae that obtain value $\mathbf{1}$ in all interpretations in all BL-algebras. The next step is to fix the axiom schemata and the rules of inference: a well-formed formula is a theorem if it is either an axiom or it is obtained recursively from axioms by using rules of inference finitely many times. Completeness of the logic means that tautologies and theorems coincide; Classical Sentential Logic and Basic Fuzzy Sentential Logic are complete logics.

In Pavelka's Fuzzy Sentential Logic [16, 17, 18] the situation is somewhat different. We start by fixing a set of truth values with a certain type of algebraic structure -in Pavelka's own approach as well as in Hájek's work [13] this structure is the standard $\mathrm{MV}$-algebra $\mathcal{C} L$ on the real unit interval while in [21] the structure is a more general (but fixed!) injective MV-algebra $L$. In this brief review of Pavelka's Fuzzy Logic we follow [21].

\subsection{Language}

Consider a zero order language $\mathcal{C} F$ with a set of countably infinitely many propositional variables $\mathrm{p}, \mathrm{q}, \mathrm{r}, \cdots$, and a set of truth constants $\{\mathbf{a} \mid a \in L\}$ corresponding to elements in the set $L$ of truth values. As proved in [13], if the set of truth values is the whole real interval $[0,1]$ then it is enough to include truth constants corresponding to rationals $r \in[0,1]$; Hájek calls this modification of Pavelka's Logic Rational Pavelka Logic. In Boolean Logic truth constants reduce to the truth constants $\perp$ and $T$. Propositional variables and truth constants constitute the set $\mathcal{C} F_{0}$ of atomic formulae. The elementary logical connectives are implication 'imp' and conjunction 'and'. The set of all well formed formulae (wffs) is obtained in the natural way: atomic formulae are wffs and if $\alpha, \beta$ are wffs, then ' $\alpha$ imp $\beta$ ', ' $\alpha$ and $\beta$ ' are wffs. Other logical connectives are introduced as abbreviations, e.g. negation 'not' is defined by setting

$$
\operatorname{not} \alpha:=\alpha \text { imp } 0
$$

where $\mathbf{0}$ is the logical constant representing falsity, and disjunction 'or' is defined by setting

$$
\alpha \text { or } \beta:=\operatorname{not}[(\operatorname{not} \alpha) \text { and }(\operatorname{not} \beta)] \text {. }
$$

Another connective or, called weak disjunction, is an abbreviation

$$
\alpha \text { or } \beta:=(\alpha \operatorname{imp} \beta) \operatorname{imp} \beta,
$$

and as usual, an equivalence is an abbreviation

$$
\alpha \text { equiv } \beta:=(\alpha \operatorname{imp} \beta) \text { and }(\beta \operatorname{imp} \alpha) \text {. }
$$

\subsection{Semantics: valuations}

Semantics in Pavelka's Fuzzy Sentential Logic is introduced in the following way: recalling that $\mathcal{C} L$ is the standard MV-algebra, any mapping $v: \mathcal{C} F_{0} \rightarrow$
$\mathcal{C} L$ such that $v(\mathbf{a})=a$ for all truth constants a can be extended recursively into the whole $\mathcal{C} F$ by setting

$$
\begin{aligned}
& v(\alpha \operatorname{imp} \beta)=v(\alpha) \rightarrow v(\beta), \\
& v(\alpha \text { and } \beta)=v(\alpha) \odot v(\beta) .
\end{aligned}
$$

Such mappings $v$ are called valuations. The truth degree of a wff $\alpha$ is the infimum of all values $v(\alpha)$, that is

$$
\mathcal{C}^{\mathrm{sem}}(\alpha)=\bigwedge\{v(\alpha) \mid v \text { is a valuation }\}
$$

In Classical Logic, a theory is a set of wffs; they are assumed to be true, not by logical cause but by some other reason. In order to define a fuzzy theory, we take $\mathcal{T} \subseteq \mathcal{C} F$ and associate to each $\alpha \in \mathcal{C} F$ a value $\mathcal{T}(\alpha)$ determining its degree of truth. We consider valuations $v$ such that $\mathcal{T}(\alpha) \leq v(\alpha)$ for all wffs $\alpha$. If such a valuation exists, then $\mathcal{T}$ is called satisfiable and $v$ satisfies $\mathcal{T}$. We say that the corresponding formulae $\alpha$ are the special axioms of fuzzy theory $\mathcal{T}$ (called non-logical axioms of $\mathcal{T}$ in $[17,21]$ ). Then we consider values

$$
\begin{aligned}
& \mathcal{C}^{\operatorname{sem}}(\mathcal{T})(\alpha) \\
& =\bigwedge\{v(\alpha) \mid v \text { is a valuation, } v \text { satisfies } \mathcal{T}\}
\end{aligned}
$$

\subsection{Syntax: axioms and rules of inference}

The logical axioms in Pavelka's Fuzzy Logic, denoted by A, are composed of the following eleven forms of formulae (axiomatic schemata); on the right-hand side there is the value they receive in any valuation $v$ : (Ax. 1) $\alpha \operatorname{imp} \alpha$,

(Ax. 2) $\quad(\alpha \operatorname{imp} \beta) \operatorname{imp}[(\beta \operatorname{imp} \gamma) \operatorname{imp}(\alpha \operatorname{imp} \gamma)], \mathbf{1}$,

(Ax. 3) $\quad\left(\alpha_{1} \operatorname{imp} \beta_{1}\right) \operatorname{imp}\left\{\left(\beta_{2} \operatorname{imp} \alpha_{2}\right)\right.$

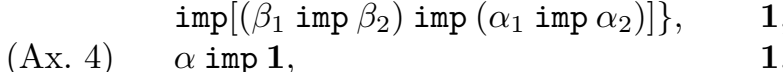

(Ax. 5) 0 imp $\alpha, \quad \mathbf{1}$,

$($ Ax. 6) $\quad(\alpha$ and $\operatorname{not} \alpha) \operatorname{imp} \beta, \quad \mathbf{1}$,

$($ Ax. 7$) \quad a, \quad a$,

(Ax. 8) $\alpha \operatorname{imp}(\beta \operatorname{imp} \alpha), \quad \mathbf{1}$,

(Ax. 9) (1 imp $\alpha) \operatorname{imp} \alpha, \quad \mathbf{1}$,

(Ax. 10) $[(\alpha \operatorname{imp} \beta) \operatorname{imp} \beta] \operatorname{imp}[(\beta \operatorname{imp} \alpha) \operatorname{imp} \alpha], \mathbf{1}$,

(Ax. 11) (not $\alpha$ imp not $\beta) \operatorname{imp}(\beta \operatorname{imp} \alpha), \quad 1$.

Thus all the axiomatic schemata $\delta$ in (Ax. 1)(Ax. 6) and (Ax. 8)-(Ax. 11) are 1-tautologies, that is $\mathcal{C}^{\operatorname{sem}}(\delta)=\mathbf{1}$ and, for axioms (Ax. 7), $\mathcal{C}^{\operatorname{sem}}(\mathbf{a})=a \in L$. A fuzzy rule of inference is a scheme

$$
\frac{\alpha_{1}, \cdots, \alpha_{n}}{r^{\mathrm{syn}}\left(\alpha_{1}, \cdots, \alpha_{n}\right)} \quad, \quad \frac{a_{1}, \cdots, a_{n}}{r^{\operatorname{sem}}\left(a_{1}, \cdots, a_{n}\right)}
$$

where the wffs $\alpha_{1}, \cdots, \alpha_{n}$ are premises and the wff $r^{\operatorname{syn}}\left(\alpha_{1}, \cdots, \alpha_{n}\right)$ is the conclusion. The values $a_{1}, \cdots, a_{n}$ and $r^{\operatorname{sem}}\left(a_{1}, \cdots, a_{n}\right) \in L$ are the corresponding truth values. The mappings 
$r^{\text {sem }}: L^{n} \rightarrow L$ are left continuous, i.e.

$$
\begin{aligned}
& r^{\mathrm{sem}}\left(a_{1}, \cdots, \bigvee_{j \in \Gamma} a_{k_{j}}, \cdots, a_{n}\right) \\
& =\bigvee_{j \in \Gamma} r^{\operatorname{sem}}\left(a_{1}, \cdots, a_{k_{j}}, \cdots, a_{n}\right)
\end{aligned}
$$

holds for all $1 \leq k \leq n$. Moreover, the fuzzy rules are required to be sound in the sense that

$$
r^{\operatorname{sem}}\left(v\left(\alpha_{1}\right), \cdots, v\left(\alpha_{n}\right)\right) \leq v\left(r^{\operatorname{syn}}\left(\alpha_{1}, \cdots, \alpha_{n}\right)\right)
$$

holds for all valuations $v$.

The following are examples of fuzzy rules of inference, denoted by a set $\mathrm{R}$ in [18]:

Generalized Modus Ponens:

$$
\frac{\alpha, \alpha \operatorname{imp} \beta}{\beta}, \frac{a, b}{a \odot b}
$$

a-Consistency testing rules:

$$
\underline{\mathbf{a}} \quad, \quad \underline{b}
$$

where $\mathbf{a}$ is a truth constant and $c=\mathbf{0}$ if $b \leq a$ and $c=\mathbf{1}$ otherwise.

a-Lifting rules:

$$
\frac{\alpha}{\mathbf{a} \operatorname{imp} \alpha} \quad, \quad \frac{b}{a \rightarrow b}
$$

where $\mathbf{a}$ is a truth constant.

Rule of Bold Conjunction:

$$
\frac{\alpha, \beta}{\alpha \text { and } \beta} \quad, \quad \frac{a, b}{a \odot b}
$$

It is easy to see that also a Rule of Bold Disjunction (not included in the list of Pavelka)

$$
\frac{\alpha, \beta}{\alpha \operatorname{or} \beta} \quad, \quad \frac{a, b}{a \oplus b}
$$

is a rule of inference in Pavelka's sense [23].

An R-proof (by Pavelka) $w$ of a wff $\alpha$ in a fuzzy theory $\mathcal{T}$ is a finite sequence

$$
\begin{array}{ccc}
\alpha_{1} & , & a_{1} \\
\vdots & & \vdots \\
\alpha_{m} & & a_{m}
\end{array}
$$

where

(i) $\alpha_{m}=\alpha$,

(ii) for each $i, 1 \leq i \leq m, \alpha_{i}$ is a logical axiom or a special axiom of a fuzzy theory $\mathcal{T}$, or there is a fuzzy rule of inference and well formed formulae $\alpha_{i_{1}}, \cdots, \alpha_{i_{n}}$ with $i_{1}, \cdots, i_{n}<i$ such that $\alpha_{i}=$ $r^{\operatorname{syn}}\left(\alpha_{i_{1}}, \cdots, \alpha_{i_{n}}\right)$,

(iii) for each $i, 1 \leq i \leq m$, the value $a_{i} \in L$ is given by

$$
a_{i}=\left\{\begin{array}{cc}
\begin{array}{l}
a \\
\mathbf{1}
\end{array} \quad \text { if } \alpha_{i} \text { is the truth constant } \mathbf{a}, \\
\quad \text { if } \alpha_{i} \text { is a logical axiom in } \mathrm{A}, \\
\mathcal{T}\left(\alpha_{i}\right) \\
\text { if } \alpha_{i} \text { is a special axiom of } \mathcal{T}, \\
r^{\mathrm{sem}}\left(a_{i_{1}}, \cdots, a_{i_{n}}\right) \\
\text { if } \alpha_{i}=r^{\mathrm{syn}}\left(\alpha_{i_{1}}, \cdots, \alpha_{i_{n}}\right) .
\end{array}\right.
$$

The value $a_{m}$ is called the degree of the R-proof $w$. Since a wff $\alpha$ may have various R-proofs with different degrees, we define the provability degree of a formula $\alpha$ to be the supremum of all such values, i.e.,

$\mathcal{C}^{\operatorname{syn}}(\mathcal{T})(\alpha)$

$=\bigvee\left\{a_{m} \mid w\right.$ is an $\mathrm{R}$-proof for $\alpha$ in fuzzy theory $\left.\mathcal{T}\right\}$.

In particular, $\mathcal{C}^{\operatorname{syn}}(\mathcal{T})(\alpha)=\mathbf{0}$ means that either $\alpha$ does not have any R-proof or that the degree of any R-proof $w$ of $\alpha$ is $\mathbf{0}$. A fuzzy theory $\mathcal{T}$ is consistent if $\mathcal{C}^{\operatorname{sem}}(\mathcal{T})(\mathbf{a})=a$ for all truth constants a. By [21, Proposition 94], any satisfiable fuzzy theory is consistent. Theorem 25 in [21] now states the completeness of Pavelka's Sentential Logic:

If a fuzzy theory $\mathcal{T}$ is consistent, then $\mathcal{C}^{\operatorname{sem}}(\mathcal{T})(\alpha)=\mathcal{C}^{\operatorname{syn}}(\mathcal{T})(\alpha)$ for any wff $\alpha$.

Thus, in Pavelka's Fuzzy Sentential Logic we can talk of a provability degree and a truth degree of a formula, and they always coincide.

\section{Perfect Pavelka Logic}

Since Chang's MV-algebra $\mathcal{C} C$ is an MV-algebra, it satisfies equations (15) and (16) whenever the corresponding suprema and infima exist in $\mathcal{C} C$. Assume now that the set of truth values is Chang's MValgebra $\mathcal{C} C$ and the truth constants correspond to the elements of $\mathcal{C} C$. All the concepts of Pavelka Logic remain meaningful in such a modification with the exception of the definition of completeness; $\mathcal{C}^{\operatorname{sem}}(\mathcal{T})(\alpha)$ or $\mathcal{C}^{\operatorname{syn}}(\mathcal{T})(\alpha)$ may not exists in $\mathcal{C} C$. Following [23], we call such a modification Perfect Pavelka Logic, PPL in short. We set the following

Definition 2 A fuzzy theory $\mathcal{T}$ is weakly complete if whenever $\mathcal{C}^{\operatorname{syn}}(\mathcal{T})(\alpha)$ exists then also $\mathcal{C}^{\operatorname{sem}}(\mathcal{T})(\alpha)$ exists and these two values coincide.

Due to the linearity and discrete structure of $\mathcal{C} C$ we observe that if the values $\mathcal{C}^{\operatorname{sem}}(\mathcal{T})(\alpha)$ and $\mathcal{C}^{\operatorname{syn}}(\mathcal{T})(\alpha)$ exist, then

$\mathcal{C}^{\operatorname{syn}}(\mathcal{T})(\alpha)$

$=\max \left\{a_{m} \mid a_{m}\right.$ is the value of the R-proof $w$ for $\alpha$ in fuzzy theory $\mathcal{T}\} \in \mathcal{C} C$,

$\mathcal{C}^{\operatorname{sem}}(\mathcal{T})(\alpha)$

$=\min \{v(\alpha) \mid v$ is a valuation, $v$ satisfies $\mathcal{T}\} \in \mathcal{C} C$.

Thus, there is an R-proof $w$ for $\alpha$ in the fuzzy theory $\mathcal{T}$ with provability degree $a=a_{m}=\mathcal{C}^{\operatorname{syn}}(\mathcal{T})(\alpha)$ and a valuation $v$ that satisfies $\mathcal{T}$ and $b=v(\alpha)=$ $\mathcal{C}^{\operatorname{sem}}(\mathcal{T})(\alpha)$. In such cases we write $\mathcal{T} \vdash_{a} \alpha$ and $\mathcal{T} \models_{b} \alpha$. (Notice that the symbol $\vdash_{a}$ denotes that the provability degree is exactly $a$, not at least $a$. Thus the existence of a proof of $\alpha$ with value $a$ is a weaker condition than $\mathcal{T} \vdash_{a} \alpha$.) Our aim is to prove that if a formula $\alpha$ is provable at a degree $a \in \mathcal{C} C$ in a consistent fuzzy theory $\mathcal{T}$, then its truth degree is $a$. 
REMARK 3 In PPL, it suffices to introduce just one truth constant, $\mathbf{f}$ or $\mathbf{t}$; all other constants from Chang's MV-algebra can be derived. This situation differs from that of Rational Pavelka Logic where infinitely many truth constants are needed.

\subsection{Axioms and rules of inference of Perfect Pavelka Logic}

Axioms of Perfect Pavelka Logic (as is the original Pavelka approach, denoted by A) are the schemata (Ax. 1)-(Ax. 11) and the following

(Ax. 12) $\quad[(\alpha$ or $\alpha)$ and $(\alpha$ or $\alpha)]$ equiv $[(\alpha$ and $\alpha)$ or $(\alpha$ and $\alpha)], \quad \mathbf{1}$,

$(\mathrm{Ax} .13) \quad[\alpha$ or $(\operatorname{not} \alpha$ and $\beta)]$ $\operatorname{imp}[(\alpha \operatorname{imp} \beta) \operatorname{imp} \beta], \quad \mathbf{1}$,

(Ax. 14) a imp b,

$1, b$
$a \rightarrow b$ where $\alpha, \beta$ are wffs and $\mathbf{a}, \mathbf{b}$ are truth constants. In Chang's MV-algebra $\mathcal{C} C$ the axioms (Ax. 12) obtain value $\mathbf{1}$ in all valuations. In any MV-algebra the axioms (Ax. 13) obtain value 1 in all valuations, and axioms (Ax. 14), called book-keeping axioms, obtain a value $a \rightarrow b$. The rules of inference are those of the original Pavelka Logic and the Rule of Bold Disjunction (all together again denoted by R); they satisfy isotonicity and soundness in any MV-algebra, thus in Chang's MV-algebra $\mathcal{C} C$, too. On the basis of the choice of the axioms and by soundness condition of rules of inference, a satisfiable fuzzy theory $\mathcal{T}$ is sound; if $\mathcal{T} \vdash_{a} \alpha$ and $\mathcal{T} \models_{b} \alpha$ exist, then $a \leq b$.

\subsection{Completeness of Perfect Pavelka Logic}

Recall that a fuzzy theory $\mathcal{T}$ is consistent if $\mathcal{C}^{\operatorname{sem}}(\mathcal{T})(\mathbf{a})=a$ for all truth constants a, otherwise it is inconsistent (also called contradictory in [21]). The following results correspond to [21, Propositions 92-96].

Proposition 4 A fuzzy theory $\mathcal{T}$ is inconsistent iff $\mathcal{T} \vdash_{1} \alpha$ holds for any wff $\alpha$.

Proposition 5 A fuzzy theory $\mathcal{T}$ is inconsistent iff the following condition holds:

(C) There is a wff $\alpha$ and $\mathrm{R}$-proofs $w, w^{\prime}$ with values $a_{m}, b_{m^{\prime}}$ for $\alpha$ and not $\alpha$, respectively, such that $\mathbf{0}<a_{m} \odot b_{m^{\prime}}$.

Proposition 6 A satisfiable fuzzy theory $\mathcal{T}$ is consistent.

Proposition $7 \quad$ [23] If $\mathcal{T} \vdash_{a} \alpha$ then $\mathcal{T} \vdash_{1}(\operatorname{aimp} \alpha)$.

Since $\vdash(\alpha$ and $\beta)$ imp $\alpha$ is provable in Łukasiewicz Sentential Logic, it follows that

$$
\mathcal{T} \vdash_{1}[(\alpha \text { and } \beta) \operatorname{imp} \alpha]
$$

holds for any fuzzy theory $\mathcal{T}$; the proof is similar to that of equation (17) in [6, Proposition 6.1.4]. This fact is used in the following result.
Proposition 8 [23] If $\mathcal{T}$ is a consistent fuzzy theory and $\mathcal{T} \vdash_{a} \alpha$, then $\mathcal{T} \vdash_{0}($ not $\mathbf{a}$ and $\alpha)$.

We can now proceed exactly the same way as in [21, Chapter 3.3]. Let $\mathcal{T}$ be a fixed fuzzy theory; by defining

$$
\begin{gathered}
\alpha \equiv \beta \\
\left(\mathcal{T} \vdash_{1}(\alpha \operatorname{imp} \beta) \quad \text { and } \quad \mathcal{T} \vdash_{1}(\beta \text { imp } \alpha)\right),
\end{gathered}
$$

we obtain a congruence relation; denote by $|\alpha|$ the equivalence class of any $\alpha$ and by $\mathcal{C} F / \equiv$ the set of all equivalence classes. Then, corresponding to $[21$, Proposition 98], we have

Proposition 9 Define $|\alpha| \rightarrow|\beta|=\mid \alpha$ imp $\beta \mid$ and $|\alpha|^{*}=|\operatorname{not} \alpha|$. Then $\left\langle\mathcal{C} F / \equiv, \rightarrow,{ }^{*},|\mathbf{1}|\right\rangle$ is a Wajsberg algebra and, hence, an MV-algebra.

The Lindenbaum algebra $\mathcal{C} F / \equiv$ is, in fact, a perfect MV-algebra; moreover:

Proposition 10 Assume $\mathcal{T}$ is a consistent fuzzy theory. If $\mathcal{T} \vdash_{a} \alpha$ then $|\alpha|=|\mathbf{a}|$ in $\mathcal{C} F / \equiv$.

Proposition 10 has a consequence that $\mathcal{C F} / \equiv$ is completely determined by the truth constants, which in turn are in one-to-one correspondence with the elements of Chang's MV-algebra $\mathcal{C} C$. Therefore there is an $\mathrm{MV}$-isomorphism $\kappa:(\mathcal{C} F / \equiv) \rightarrow \mathcal{C} C$ given by $\kappa(|\mathbf{a}|)=a$, in particular $\kappa(|\mathbf{1}|)=\mathbf{1}$. This isomorphism can be proved by using the bookkeeping axioms (Ax. 14); for all truth constants $\mathbf{a}, \mathbf{b}$ a formula $(\mathbf{a}$ imp $\mathbf{b})$ is an axiom of degree $a \rightarrow b$. We observe that in consistent fuzzy theories this implies $\mathcal{T} \vdash_{a \rightarrow b}$ a impb. Indeed, assume $\mathcal{T} \vdash_{c}$ a impb, where $c>a \rightarrow b$. Since $c \leq a \rightarrow b$ iff $c \odot a \leq b$, the assumption $c>a \rightarrow \bar{b}$ implies $c \odot a \not \leq b$. Then $\mathcal{T}$ can be shown to be inconsistent by the following R-proof:

$\begin{array}{lll}\mathbf{a} \text { imp b, } & c, & \text { assumption } \\ \mathbf{a}, & a, & \text { axiom (Ax. 7) } \\ \mathbf{b}, & a \odot c, & \text { by Generalized Modus Ponens } \\ \mathbf{0}, & \mathbf{1}, & \text { by b-Consistency testing rule }\end{array}$

Therefore $\mathcal{T} \vdash_{a \rightarrow b}$ a imp b for a consistent fuzzy theory $\mathcal{T}$. In particular (recall the abbreviation of not $\alpha$ ) we have $\mathcal{T} \vdash_{a^{*}}$ not a. In the Lindenbaum algebra, $\mid \mathbf{a}$ imp $\mathbf{b}|=| \mathbf{a}|\rightarrow| \mathbf{b} \mid$ holds and, by Proposition $10, \mid \mathbf{a}$ imp $\mathbf{b}|=| \mathbf{d} \mid$, where $d=a \rightarrow b$. Thus $\kappa(\mid \mathbf{a}$ imp $\mathbf{b} \mid)=a \rightarrow b=\kappa(|\mathbf{a}|) \rightarrow \kappa(|\mathbf{b}|)$. In particular, $\kappa(|\operatorname{not} \mathbf{a}|)=\kappa\left(\left|\mathbf{a}^{*}\right|\right)=a^{*}$.

Let $\pi$ be the canonical mapping $\pi: \mathcal{C} F \rightarrow \mathcal{C} F / \equiv$. Then $\kappa \circ \pi$ is the valuation in demand; if $\mathcal{T} \vdash_{a} \alpha$ then $\kappa \circ \pi(\alpha)=\kappa(|\mathbf{a}|)=a$. In conclusion, we can write

THEOREM 11 If the provability degree of a formula $\alpha$ is a $\in \mathcal{C} C$ in a consistent fuzzy theory $\mathcal{T}$ (i.e. $\mathcal{C}^{\operatorname{syn}}(\mathcal{T})(\alpha)$ exists and equals a), then $\alpha$ is also a tautology at degree $a$, i.e. its truth degree $\mathcal{C}^{\operatorname{sem}}(\mathcal{T})(\alpha)$ exists and is equal to a. 
In particular, if $\mathcal{T} \vdash_{a} \alpha$, where $a$ is in $F$, the 'false part', we have a stronger result.

Corollary 12 Let $\mathcal{T}$ be a consistent fuzzy theory. A formula $\alpha$ has a provability degree $a \in F$ if, and only if $\alpha$ is also a tautology at degree $a$.

Notice that Theorem 11 does not imply that in a consistent fuzzy theory $\mathcal{T}$ all formulas necessarily have a truth degree or are provable at a degree; as an example let the special axioms of $\mathcal{T}$ be $\mathcal{T}(\mathbf{a}$ imp $\mathrm{p})=\mathbf{1}$ for any truth constant $\mathbf{a}, a \in F$ and a propositional variable $\mathrm{p}$. Then $\mathcal{T}$ is consistent; any valuation $v$ such that $v(\mathrm{p})=t^{n} \in T$ satisfies $\mathcal{T}$. Moreover, $\mathrm{p}$ has an R-proof with any value $f^{n} \in F$; use axiom (Ax. 7) and Generalized Modus Ponens. Hence neither $\mathcal{C}^{\operatorname{sem}}(\mathcal{T})(\mathrm{p})$ nor $\mathcal{C}^{\operatorname{syn}}(\mathcal{T})(\mathrm{p})$ exists.

More generally, if, in a consistent fuzzy theory $\mathcal{T}$ and for a formula $\alpha$, the value $\mathcal{C}^{\operatorname{syn}}(\mathcal{T})(\alpha)$ does not exist, then necessarily $\alpha$ has infinitely many Rproofs with values larger than any $f^{n} \in F$ and less than any $t^{n} \in T$ (and conversely). It is also obvious that $\mathcal{C}^{\operatorname{sem}}(\mathcal{T})(\alpha)$ does not exist if, and only if, for any natural $n$ there is a valuation $v$ satisfying $\mathcal{T}$ and $f^{n}<v(\alpha)<t^{n}$. Moreover, if $\mathcal{C}^{\operatorname{sem}}(\mathcal{T})(\alpha)$ does not exist then, by the above considerations, neither $\mathcal{C}^{\operatorname{syn}}(\mathcal{T})(\alpha)$ exists. It remains open to prove or disprove that if $\mathcal{C}^{\operatorname{sem}}(\mathcal{T})(\alpha)$ exists in $T$ then also $\mathcal{C}^{\operatorname{syn}}(\mathcal{T})(\alpha)$ exists in $T$. We assume that this is the case and formulate the following

CONJECTURE 13 Let $\mathcal{T}$ be a consistent fuzzy theory. A formula $\alpha$ has a provability degree $a \in T$ if, and only if $\alpha$ is also a tautology at degree $a$.

\section{What can and what cannot be expressed in Rational or Perfect Pavelka Logic}

One of the arguments for (some) fuzzy logics is that they admit to explain the Sorites Paradox [13]. A fuzzy statement like "this person is not bald" may be true at the beginning; then its truth degree may decrease by small decrements and after many repetitions, it may become false. The gradual true with a dense set of truth values admits to model this phenomenon and overcome a paradox from Classical Logic. Łukasiewicz logic, whose semantics is based on the standard MV-algebra, is one of the fuzzy logics where this can be explained.

In contrast to this, Chang's MV-algebra does not allow to explain the Sorites Paradox. If the statement "this person is not bald" is true at the beginning and its truth degree decreases by small decrements (infinitesimals), it never reaches the value false; the truth degree remains infinitesimally close to 1 unless it makes a "big jump" into the degrees infinitesimally close to 0 .

Despite this difference, Chang's MV-algebra and infinitesimals have their role in modeling human reasoning. E.g., let us consider the task of traveling to an airport. A truth degree should express how convenient the chosen way is. We may optimize the way in terms of cost, time, choosing the route, etc. However, all these improvements are negligible in comparison with the crucial question whether we catch our flight or not. Any number of "small" advantages cannot compensate the big disadvantage when we miss the flight. Thus their representation by infinitesimals is adequate. This does not mean that - as soon as we choose only from options in which we catch the flight - we should ignore these small contributions, e.g., saving cost by choosing among several sufficiently fast options.

Thus the adequacy of both models depends on the specifics of the situation. Sometimes "many small contributions may compensate a big change", sometimes not. More exactly, the use of infinitesimals admits to express that some changes (of a truth value) are nonzero, but infinitely many times smaller than others.

The two semantics studied here are two extremes: The standard MV-algebra does not admit any infinitesimals, while the Chang's MV-algebra contains only infinitesimals (and their duals). There are more general MV-algebras which combine infinitesimals and non-infinitesimals. Their complete characterization follows from [14, 5], see also [4]. The semantics based on such MV-algebras could describe two types of changes of truth values - "big" ones which may model the Sorites Paradox and infinitesimal ones for which this paradox applies (as in Classical Logic). We expect that a Pavelka-style logic could be based on general MV-algebras as well.

A typical example is the interval $[(0,0),(1,0)]$ in the lexicographical product $M=\mathbb{Q} \times{ }_{\text {lex }} \mathbb{Z}$, where $\mathbb{Q}$, resp. $\mathbb{Z}$, is the set of all rational, resp. integer, numbers. The MV-algebraic operations on $M$ are defined as follows:

$$
\begin{aligned}
\mathbf{0} & =(0,0), \\
\mathbf{1} & =(1,0), \\
(q, n)^{*} & =(1-q,-n), \\
(p, k) \oplus(q, n) & =\min ((1,0),(p+q, k+n)) .
\end{aligned}
$$

The set $\{(0, n) \mid n \in \mathbb{Z}, n \geq 0\}$ is closed under $\oplus$, its elements are infinitesimals. On the other hand, elements of the form $(q, n), q>0$, are not infinitesimals; the sum of $\lceil 1 / q\rceil$ such elements is $\mathbf{1}=(1,0)$. The former elements have properties described in Perfect Pavelka Logic. The latter elements have properties known from Rational Pavelka Logic. We expect that the combination of both approaches could further extend the possibility of modelling of the human reasoning based on graduate truth values.

Acknowledgement The authors acknowledge the support by the Ministry of Education of the Czech Republic under Project RVO13000. 


\section{References}

[1] Belluce, P.L., Di Nola, A., and Gerla, B.: Perfect MV-algebras and their Logic. Applied Categorical Structures 15 (2007) 135-151.

[2] Blok, W.J. and Pigozzi, D.: Algebraizable Logics. Memoirs of the American Mathematical Society 77 (1989) Number 396.

[3] Chang, C.C.: Algebraic analysis of manyvalued logics. Transactions of the American Mathematical Society 88 (1958), 476-490.

[4] Cignoli, R., D'Ottaviano I.M.L., and Mundici, D.: Algebraic Foundations of many-valued Reasoning. Trends in Logic, vol. 7, Kluwer Academic Publishers, Dordrecht (2000).

[5] Di Nola, A., Lettieri, A.: Equational Characterization of all Varieties of MV-algebras. J. of Algebra 221 (1999) 463-474.

[6] Di Nola, A. and Leustean, I.: Łukasiewicz Logic and MV-Algebras. In: Handbook of Mathematical Fuzzy Logic II (eds. Cintula, P., Noguera, C., and Hájek, P.) Studies in Logic 36 (2011) 469-583.

[7] Esteva, F., Gispert, J., Godo, L., and Noguera, C.: Adding truth-constants to logics of continuous t-norms: Axiomatization and completeness results. Fuzzy Sets and Systems 158 (2007) 597-618.

[8] Esteva, F., Godo, L., and Noguera, C.: On completeness results for Predicate Łukasiewicz, Product, Gödel and Nilpotent Minimum Logics Expanded with Truth-constants. Mathware \& Soft Computing 14 (2007) 233-246.

[9] Esteva, F., Godo, L., and Noguera, C.: Firstorder t-norm based fuzzy logics with truthconstants: Distinguished semantics and completeness properties. Annals of Pure and Applied Logic 161 (2009) 185-202.

[10] Esteva, F., Godo, L., and Noguera, C.: Expanding the propositional logic of a t-norm with truth-constants: completeness results for rational semantics. Soft Computing 14 (2010) 273-284.

[11] Gerla, G.: Fuzzy Logic: Mathematical Tools for Approximate Reasoning. Springer-Verlag (2001).

[12] Goguen, J. A.: The logic of inexact concepts. Synthese 19 (1968/69) 325-373.

[13] Hájek, P.: Metamathematics of Fuzzy Logic. Kluwer (1998).

[14] Komori, Y.: Super-Łukasiewicz propositional logic. Nagoya Math. J. 84 (1981) 119-133.

[15] Novák, V.: First-order fuzzy logic. Studia Logica 46 (1987) 87-109.

[16] Pavelka, J.: On fuzzy logic I. Zeitsch. f. Math. Logik 25 (1979) 45-52.

[17] Pavelka, J.: On fuzzy logic II. Zeitsch. f. Math. Logik 25 (1979) 119-134.

[18] Pavelka, J.: On fuzzy logic III. Zeitsch. f.
Math. Logik 25 (1979) 447-464.

[19] Savický, P., Cignoli, R., Esteva. F, Godo, L., and Noguera, C.: On product logic with truthconstants. Journal of Logic and Computation 16 (2006) 205-225.

[20] Suppes, P.: Introduction to Logic. Van Nostrand (1957).

[21] Turunen, E.: Mathematics Behind Fuzzy Logic. Springer-Verlag (1999).

[22] Turunen, E.: Well-defined fuzzy sentential logic. Mathematical Logic Quarterly. 41 (1995), 236-248.

[23] Turunen, E., Navara, M.: Perfect Pavelka Logic. Fuzzy Sets Syst., accepted. 